\title{
Ecological Tunnel for the 21st Century: A New Conception and Methodology
}

\author{
Zixin Zhang $^{{ }^{*}}$, Qinghua Lei ${ }^{1}$, Qiang Xue ${ }^{2}$ \\ ${ }^{1}$ Department of Geotechnical Engineering, School of Civil Engineering, Tongji University, Shanghai, China \\ ${ }^{2}$ State Key Laboratory of Geomechanics and Geotechnical Engineering, Institute of Rock and Soil Mechanics, Chinese \\ Academy of Sciences, Wuhan, China \\ E-mail: zxzhang@tongji.edu.cn
}

Received April 25, 2011; revised May 20, 2011; accepted May 30, 2011

\begin{abstract}
This paper presents a new conception-ecological tunnel, which is contrived to meet increasingly tough challenges in the $21^{\text {st }}$ century. Ecological tunnel refers to integrating ecological principles into tunnel design, construction and operation in order to create a balanced and sustainable tunnel-nature system. It consists of four elements: green tunneling, green lighting, green lining, and green recycling of excavated material. The conception, function and implementation of each part are elaborated in the paper. In addition, computer visualization technique is applied to simulate complicated internal conditions of an ecological tunnel.
\end{abstract}

Keywords: Ecological Tunnel, Green Tunneling, Green Lighting, Green Lining, Green Recycling, Computer Visualization

\section{Introduction}

Currently, human needs, activities and communications have increasingly stressed traffic systems and consequently produced countless artificial tunnels. The traditional modes of tunneling and measures of disposing excavated soils have yet gained unsatisfactory results. Although tunnels have brought with them numerous economic benefits to the society, they should also take responsibility for a large share of material and energy consumption, and generation of environmental emissions, both nationally and globally. In details, these adverse effects can be categorized as: consumption of electricity and other energy, cost of human resources, emission of carbon dioxide $\left(\mathrm{CO}_{2}\right)$, abandonment of excavated soils and other solid waste, and disturbance of ecological equilibrium. Especially, the current tunneling activities have often been merely focusing on the excavation process rather than the balance between tunnels and nature. These phenomena, combined with the advent of scientific knowledge on ecosystems, make a request for new strategies to construct tunnels, and then the conception of ecological tunnel is put forward.

During the past several decades, ecologists have proposed a new method of urban planning and construction, based on the concept of ecosystem [1]. By the late 1980s, this ecosystem-based approach has been advocated by many scientists, engineers, managers and organizations, such as Agee and Johnson (1988), Kibert (2005) and US Department of Energy (USDE, 2009) [2-5]. Increasing awareness of environmental issues and negative effects from tunnel construction and operation coupled with pressures from society has led us to establish a new concept which is named as "ecological tunnel" or "eco-tunnel". The eco-tunnel system would be a potential guidance for implementing green design and construction strategies of 21st century tunnels.

\section{Definitions and Conceptions}

\subsection{What is an Ecological Tunnel?}

Since 1935, when the term "ecosystem" was first presented by Sir Arthur Tansley (Tansley, 1935), many researchers have been trying to use this concept in various fields. The application of the ecosystem concept to tunnel is a subject concerned with studying the relationships between human groups, tunneling processes, tunnel structures and surrounding environments. Based on our imagination, ecosystem-based tunnels should have the following main characteristics:

- Eco-tunnel system is subjected to a continuous 
change in space and time.

- Eco-tunnel system is constructed by efficient tunneling and soils/rocks removing, rational installing of green linings, and pollution-free recycling of excavated soils/rocks.

- Eco-tunnel system is operated with low-carbon emission, energy-saving illumination and harmonious coexistence with the nature.

There is an organized network combining different parts of an ecological tunnel system, and each part performs in close connections with all others.

\subsection{Definition of Ecological Tunnel}

Based on the aforementioned characteristics of an ecological tunnel, its definition can be given as following:

Ecological tunnel is the integrating scientific knowledge of ecology and energy-conservation within tunnel design, construction and operation towards the major goal of striking a careful balance between human beings, tunnels and nature, which is achieved by the collaboration of green tunneling, green lighting, green lining and green recycling of excavated material.

The realization of an eco-tunnel system should be based on the comprehensive usage of ecological conceptions, economic and political knowledge, power-saving technologies, geotechnical theories, and even botanical techniques.

\section{Ecological Tunnel System}

Ecological tunnel system is composed of four parts: green tunneling, green lighting, green lining, and green recycling of excavated material.

\subsection{Green Tunneling}

Tunneling is an art. Over time, numerous advanced methodologies and techniques of tunneling have been deliberately devised and developed. These techniques have served well in meeting demands of desired progress and limited time with low accident rates and few tunneling problems. Chinese designers, scientists and engineers, who have successfully accomplished a large number of tunneling projects, are currently concentrating on new challenging projects and also attempting several innovative ideas to satisfy future requirements.

In response to rapid pace of national and global development, there has been a pressing need of new tunnels for congested traffic systems and massive underground facilities. Tunneling, in virtue of its numerous excellent qualities, has gradually been proved to be an attractive approach in creating underground spaces for transport and utility networks. However, the modern tunneling modes still inevitably cause a variety of problems, including third party impact, environmental disruption and energy wastage. For instance, tunnel construction by a slurry shield beneath groundwater table will inevitably cause the drawdown of groundwater level, the pollution of surrounding soils and even the influence to residents nearby.

The concept of green tunneling is just proposed to settle these matters and the mode consists of three features:

- The decision of green tunneling method should be concerned with not only economic and technological feasibility, but also political, ethical, sociological and environmental effects.

- Green tunneling should technically achieve: low power dissipation, low emission of greenhouse gas, low noise pollution, low material consumption, low economic expenditure, low environmental disturbance and low manpower cost.

Green tunneling should be accomplished under effective management for the sake of reducing material, energy and human resource consumption.

\subsection{Green Lighting}

Green lighting system comprises collecting, converting, transmitting, storing and illuminating parts. In this system, the input is natural light including all visible wavelengths, and the output is the redistributed sunlight which, however, must comply with traffic regulations. The functions and collaborations of these subsystems are illustrated in Figure1.

- The collecting subsystem is in charge of gaining sunlight that will be transmitted into the converting subsystem.

- The converting subsystem is a device of photoelectric conversion which can transfer the collected sunlight into electric energy.

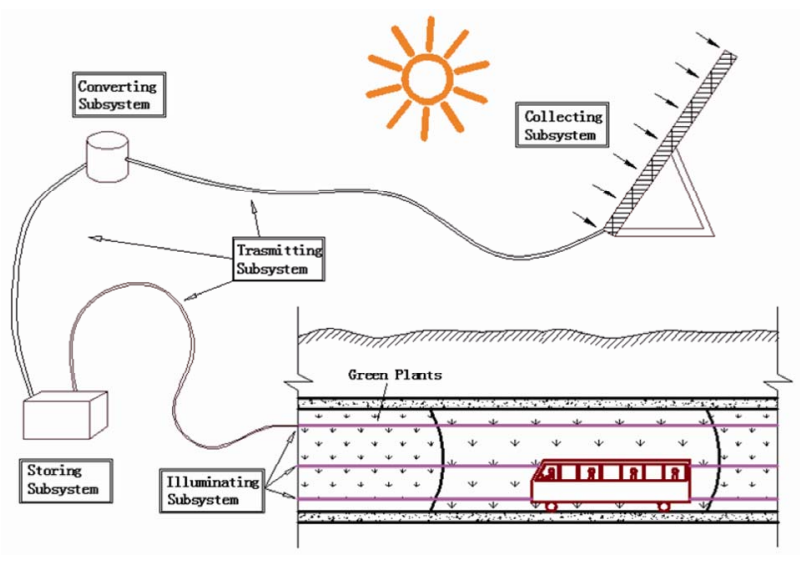

Figure 1. Green Lighting System. 
- The collecting subsystem is in charge of gaining sunlight that will be transmitted into the converting subsystem.

- The converting subsystem is a device of photoelectric conversion which can transfer the collected sunlight into electric energy.

- The storing subsystem, just as its name implies, is responsible for storing and supplying electric energy.

- The illuminating subsystem will reproduce the sunlight using optical fibers to provide an agreeable environment for driving and plants growing.

In addition, it is the transmitting subsystem that connects all other subsystems closely and efficiently

\subsection{Green Lining}

Designing and constructing the green lining system should overcome the following main and difficult points:

- Appropriate growth mediums for green plants being cultivated in tunnels should be selected, and the technique of fixing mediums on linings should also be mastered;

- Green plants with good quality of living in tunnels must be chosen from thousands kinds of plants;

- Requirements of the selected plant, including light intensity, wave lengths, temperature and humidity, should be tested systemically;

- The approach of creating such environment and guaranteeing nutrient supply should also be devised;

A dynamic adjusting function of the system should be additionally embodied in order to provide an optimal natural circumstance timely and efficiently for plants and human beings.

\subsection{Green Recycling of Excavated Material}

Soils/Rocks produced by industrial practice can be described as a sink of pollutants and a source of pollution with the quality of delivering pollutants into groundwater, into food chains and into human bodies, which would undoubtedly risk people's health. Green recycling in tunnel construction is the very solution for this issue and is achieved with three main steps, successively.

- Assessment of excavated soils/rocks

The concentration and distribution of major pollutants contained in excavated samples, such as heavy metals and some organic substances, are tested comprehensively in the laboratory. Then results are compared with the standard criteria in order to determine the contaminated state of excavated soils/rocks.

- Washing of contaminated soils/rocks

If the experimental report of samples indicates that excavated soils/rocks have been contaminated, a series of effective separation and cleaning measures would be necessary before recycling. A variety of pollution treatments that use physical and/or chemical techniques have been invented and devised by many environmental experts. These measures have been widely used in different regions and demonstrated their amazing superiorities.

- Ruse of washed soils/rocks

Treated soils or rocks would be of great availability in many aspects, such as agricultural planting, embankment fill, urban greening, land reclamation and so forth; however, the decision on applications should be made flexibly. Furthermore, a multidisciplinary approach involving geotechnical, environmental and computer database techniques is necessary to ensure the excavated material could be well understood and managed in order to optimize their values.

The detailed procedure of green recycling is illutrated in Figure 2.

\section{Simulation and visualization}

The driving conditions, lighting effects and plants growth can be simulated meticulously using computer visualization techniques. Figure 3 shows the result of an initial 3D-model of an ecological tunnel which is completed on the platform of AutoCAD 2009. In this model, the tunnel structure, optical fibers, green plants, and

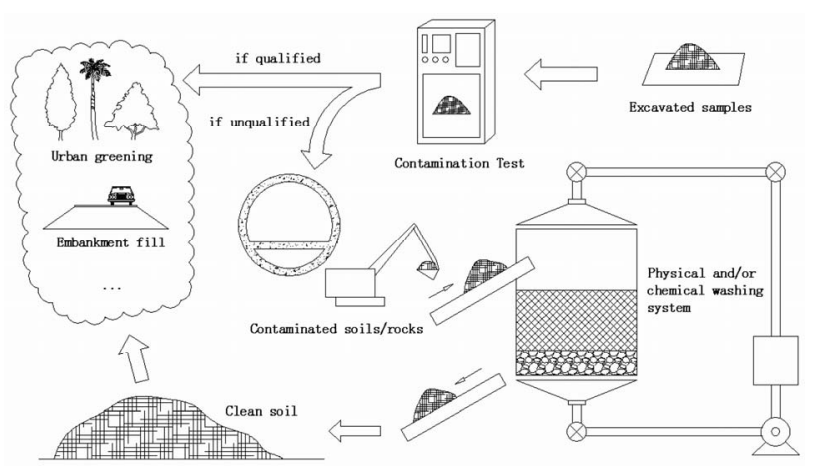

Figure 2. Green recycling of excavated soils/rocks.

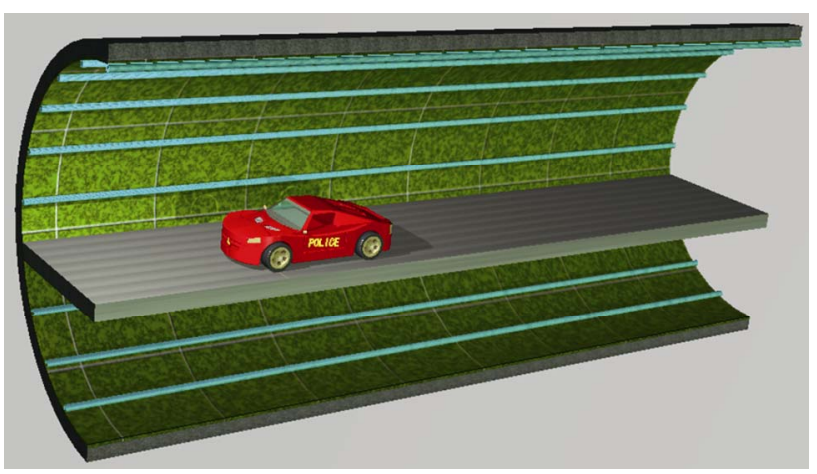

Figure 3. Visualization of an ecological tunnel. 
driving condition are visualized vividly.

\section{Conclusions}

Ecological tunnel, a new tunnel mode involving green tunneling, green lining, green lighting and green recycling of excavated soils/rocks, is created in the paper. The implementation of the eco-tunnel system, which should be based on the combination of geotechnical, environmental, botanical and computer techniques, is undoubtedly endowed with promising future in facing increasing demands and challenges of the $21^{\text {st }}$ century.

\section{References}

[1] A. Singh, G. Berghorn, S. Joshi and M. Syal, "Review of Life-Cycle Assessment Applications in Building Construction,” Journal of Architectural Engineering, Vol. 11, No. 1, 2010, pp. 25-34.
[2] C.-N. Chen, Y.-Y. Chen, A. J.-W. Whang and L.-H. Chen, "Design and Evaluation of Natural Light Guiding System in Ecological Illumination of Traffic Tunnel," Proceedings of Security Professionals Information Exchange, 7423, 2009, pp. 1-9.

[3] C. R. De Kimpe and J. L. Morel, "Urban Soil Management: A Growing Concern,” Soil Science, Vol. 165, No. 1, 2000, pp. 31-40.

doi:10.1097/00010694-200001000-00005

[4] Georgios E. Pavlikakis, Vassilios A. Tsihrintzis. "Ecosystem Management: A Review of a New Concept and Methodology," Water Resources Management, Vol. 14, No. 4, 2000, pp. 257-283. doi:10.1023/A:1008139011867

[5] S. G. Lu and S. Q. Bai. "Contamination and potential mobility assessment of heavy metals in urban soils of Hangzhou, China relationship with different land uses," Environmental Earth Sciences, Vol. 60, No. 7, 2010, pp.1481-1490. doi:10.1007/s12665-009-0283-2 